\title{
On the influence of non-thermal pressure on the mass determination of galaxy clusters
}

\author{
T. F. Laganá, R. S. de Souza, and G. R. Keller
}

\begin{abstract}
Universidade de São Paulo, Instituto de Astronomia, Geofísica e Ciências Atmosféricas, Departamento de Astronomia, Rua do Matão 1226, Cidade Universitária, 05508-090, São Paulo, SP, Brazil

e-mail: tflagana@astro.iag.usp.br
\end{abstract}

Received 16 February 2009 / Accepted 10 November 2009

\begin{abstract}
Aims. Given that in most cases just thermal pressure is taken into account in the hydrostatic equilibrium equation to estimate galaxy cluster mass, the main purpose of this paper is to consider the contribution of all three non-thermal components to total mass measurements. The non-thermal pressure is composed by cosmic rays, turbulence and magnetic pressures.

Methods. To estimate the thermal pressure we used public XMM-Newton archival data of five Abell clusters to derive temperature and density profiles. To describe the magnetic pressure, we assume a radial distribution for the magnetic field, $B(r) \propto \rho_{\mathrm{g}}^{\alpha}$. To seek generality we assume $\alpha$ within the range of 0.5 to 0.9 , as indicated by observations and numerical simulations. Turbulent motions and bulk velocities add a turbulent pressure, which is considered using an estimate from numerical simulations. For this component, we assume an isotropic pressure, $P_{\text {turb }}=\frac{1}{3} \rho_{\mathrm{g}}\left(\sigma_{\mathrm{r}}^{2}+\sigma_{\mathrm{t}}^{2}\right)$. We also consider the contribution of cosmic ray pressure, $P_{\mathrm{cr}} \propto r^{-0.5}$. Thus, besides the gas (thermal) pressure, we include these three non-thermal components in the magnetohydrostatic equilibrium equation and compare the total mass estimates with the values obtained without them.

Results. A consistent description for the non-thermal component could yield a variation in mass estimates that extends from $10 \%$ to $\sim 30 \%$. We verified that in the inner parts of cool core clusters the cosmic ray component is comparable to the magnetic pressure, while in non-cool core clusters the cosmic ray component is dominant. For cool core clusters the magnetic pressure is the dominant component, contributing more than $50 \%$ of the total mass variation due to non-thermal pressure components. However, for non-cool core clusters, the major influence comes from the cosmic ray pressure that accounts for more than $80 \%$ of the total mass variation due to non-thermal pressure effects. For our sample, the maximum influence of the turbulent component to the total mass variation can be almost $20 \%$. Although all of the assumptions agree with previous works, it is important to notice that our results rely on the specific parametrization adopted in this work. We show that this analysis can be regarded as a starting point for a more detailed and refined exploration of the influence of non-thermal pressure in the intra-cluster medium (ICM).
\end{abstract}

Key words. magnetic fields - magnetohydrodynamics (MHD) - X-rays: galaxies: clusters - galaxies: clusters: individual: Abell

\section{Introduction}

Clusters of galaxies are powerful tools for investigations of cosmological interests. The evolution of the mass function of a cluster is highly sensitive to cosmological models since the matter density controls the rate at which structures grow (Voit 2005). In order to use clusters of galaxies as observational probes of dark energy in the Universe and to investigate the structure formation history including baryonic hydrodynamics, the non-thermal contribution must be well understood and quantified.

$\mathrm{X}$-ray data are one of the methods used most often to determine the mass distribution of clusters of galaxies. To do so, hydrostatic equilibrium is usually assumed, and the observed gas density and temperature profiles are used to compute the thermal pressure. In most cases, only the gas (thermal) pressure is considered to evaluate the dynamical masses of galaxy clusters (e.g., David et al. 1995; White \& Fabian 1995; Finoguenov et al. 2001; Reiprich \& Böhringer 2002). However, there is also a nonthermal pressure $\left(P_{\mathrm{NT}}\right)$, composed by the magnetic $\left(P_{\mathrm{B}}\right)$, turbulent $\left(P_{\text {turb }}\right)$ and cosmic ray $\left(P_{\mathrm{cr}}\right)$ components that is frequently assumed to be negligible and thus ignored. As a consequence of this, today the accuracy of the hydrostatic mass estimates is limited by the non-thermal pressure from these components.
Despite the difficulty to reliably calculate the small-scale properties of the magnetic field, the existence of intra-cluster magnetic fields is well established from the studies of the rotation measure of polarized radio frequencies and synchrotron emission from diffuse sources (e.g., Andernach et al. 1988; Giovannini et al. 1993; Taylor et al. 1994, 2002; Govoni \& Feretti 2004). More recently, another indication that the intracluster medium (ICM) is permeated by a magnetic field came from the studies of X-ray cold fronts (sharp discontinuities in $\mathrm{X}$-ray surface brightness profile and temperature, Markevitch \& Vikhlinin 2007). In these cases, a parallel magnetic field can suppress transport processes in the ICM, making it difficult to mix different gas phases during a cluster merger. Even a very weak magnetic field can effectively inhibit transport processes such as thermal conduction and the settling of heavy ions (Sarazin 1986; Soker \& Sarazin 1990).

Strong magnetic fields can make a significant contribution to the gas pressure support (Loeb \& Mao 1994), contributing with a non-thermal component in the magnetohydrostatic equilibrium equation (Dolag et al. 2001b; Vogt \& Enßlin 2005). Indeed, magnetic fields as high as $10-100 \mu \mathrm{G}$ were found in Hydra A (Taylor \& Perley 1993), Cygnus A (Dreher et al. 1987) and in 3C 295 (Perley \& Taylor 1991). Moreover, Dolag et al. (1999) 
performed numerical simulations and found that even clusters with an overall small magnetic field can be penetrated partially by regions of high magnetic fields.

Although on average the magnetic pressure in simulations is much smaller than the thermal pressure $(\sim 5 \%$, Dolag \& Schindler 2000), there are domains of high magnetic fields approaching or sometimes even exceeding equipartition with the thermal energy. Previous studies (Dolag et al. 2001a; Colafrancesco \& Giordano 2007, among others) have analyzed the effects of the magnetic pressure in simulated galaxy clusters.

Magnetic fields and turbulence are possibly related to one another. It seems plausible that the turbulent motions in the ICM can maintain the magnetic field by converting kinetic energy into magnetic energy (Sánchez-Salcedo et al. 1999). The observed small-scale turbulence in the ICM can be due to bulk velocities and ongoing merger of substructures. Gas turbulence on small scales can also be driven directly by motions of galaxies, as for instance by jets and bubbles from the active galactic nuclei (AGN, Churazov et al. 2002), although the latter may be confined to the inner regions of the cluster (Lau et al. 2009). The presence of random gas motion can also contribute to the pressure support in clusters of galaxies.

The chaotic nature of the ICM magnetic field makes it difficult for energetic particles to scape from the cluster, and thus cosmic-ray protons would be confined for timescales exceeding the Hubble time. The electron cosmic rays, on the other hand, have collisional and radiative lives much shorter. Thus, since the ICM is permeated by significant magnetic fields, one would expect the cosmic ray pressure to have some relevance in its support against gravity.

To consider deviations from the standard assumptions in computing cluster total mass, the main aim of this work is to analyze the effects of non-thermal pressure, that is to take into account magnetic, turbulent and cosmic ray components. Hydrostatic masses were derived using X-ray observational data for five Abell clusters: A496, A2050, A1689, A2667 and A2631. To do so, we use temperature and density profile fits from a previous work (Laganá et al. 2008) and we introduce the $P_{\mathrm{NT}}$ contribution in the magnetohydrostatic equilibrium equation. For these five clusters, we compare masses determined considering nonthermal pressure $\left(M_{\mathrm{NTP}}(r)\right)$ with their hydrostatic values $(M(r))$.

The paper is organized as follows. We show the data sample in Sect. 2. The non-thermal components are described in Sect. 3. In this section we describe the structure of the intra-cluster magnetic field, the turbulence in the ICM and the cosmic ray component. In Sect. 4, we present the method of determining the cluster mass, including the effects of the $P_{\mathrm{NT}}$. Our results, as well as a discussion of them are presented in Sect. 5 and our conclusions in Sect. 6.

\section{Data sample}

The objects in our sample are within the redshift range of $0.03<$ $z<0.3$ and are drawn from a set of Abell clusters with available data in the XMM-Newton public archive. These clusters were previously analyzed by Laganá et al. (2008), who derived the density profiles fit parameters to compute the total mass. Although we have not used the object morphology as a criterion for the cluster selection, all these clusters except A2631 have apparently symmetric X-ray isophotes, suggesting that they are relatively relaxed. The deviations in the surface brightness profile of A2631, although clearly present, are not very large and do not invalidate the assumption of spherical symmetry. However, we
Table 1. General cluster properties.

\begin{tabular}{ccccc}
\hline \hline Cluster & $\begin{array}{c}\text { RA } \\
(\mathrm{J} 2000)\end{array}$ & $\begin{array}{c}\text { Dec } \\
(\mathrm{J} 2000)\end{array}$ & $z$ & $\begin{array}{c}r_{500} \\
h_{70}^{-1} \mathrm{kpc}\end{array}$ \\
\hline A496 & 043337.1 & -131446 & 0.033 & 1480 \\
$\mathrm{~A} 2050$ & 151621.6 & +000559 & 0.1183 & 2172 \\
$\mathrm{~A} 1689$ & 131134.2 & -012156 & 0.1823 & 1785 \\
$\mathrm{~A} 2667$ & 235147.1 & -260018 & 0.23 & 2153 \\
A2631 & 233739.7 & +001737 & 0.273 & 1976 \\
\hline
\end{tabular}

are aware of the fact that it may affect total mass reconstruction, accounting for underestimated mass determinations (Piffaretti \& Valdarnini 2008).

In Table 1, we present the five Abell clusters used in this work, specifying $r_{500}$, the radius inside which the mean density exceeds the critical density by a factor of 500. All masses are computed inside $r_{500}$, as it is the largest radius for which the current X-ray data require no model extrapolation (Vikhlinin et al. 2006) and is about the virial radius (Lacey \& Cole 1993).

Usually, the mass of a cluster is determined under the assumption of hydrostatic equilibrium without the contribution of non-thermal pressure. In this case, the total mass relies on the temperature and density profiles.

Satellites with better spatial resolution (like the XMM-Newton and Chandra) showed a significant difference between the surface brightness profile data points and the $\beta$-model (Cavaliere \& Fusco-Femiano 1976, 1978) at small radii for cool core clusters (Jones \& Forman 1984; Xue \& Wu 2000). Based on this observational difference, the $\beta$-model was used to describe the density distribution of non-cool core (NCC) clusters, while the Sérsic model (Pislar et al. 1997; Demarco et al. 2003) was used to characterize cool core (CC) clusters. For A2050 and A2631, the gas density $\left(\rho_{\mathrm{g}}\right)$ is described by

$\rho_{\mathrm{g}}(r)=\rho_{0}\left(1+\frac{r^{2}}{r_{\mathrm{c}}^{2}}\right)^{-3 \beta / 2}$,

where $\rho_{0}$ and $r_{\mathrm{c}}$ are the central gas density and the gas core radius, respectively. The $\beta$ parameter determines the power-law behavior at large radii. For A496, A1689 and A2667 (CC clusters) the gas density profiles were fitted by the Sérsic model given by

$\rho_{\mathrm{g}}(r)=\rho_{0}\left(\frac{r}{a}\right)^{-p^{\prime}} \exp \left[-\left(\frac{r}{a}\right)^{v}\right]$,

where $p^{\prime}=p / 2, p=1-0.6097 v+0.05563 v^{2}$ and $a=a^{\prime} 2^{1 / v}$ (Durret et al. 2005). The best-fit parameters were determined from the X-ray surface brightness profiles and were given by Laganá et al. (2008).

\section{Non-thermal components}

In this section we describe each non-thermal component considered to contribute to the pressure support.

\subsection{The magnetic profile}

In the 80s, Jaffe (1980) suggested that the intra-cluster magnetic field distribution should depend on the thermal gas density and on the distribution of massive galaxies, which means it 
would decline with cluster radius. Cluster observations provided constraints on the radial gradient of the cluster magnetic field (Brunetti et al. 2001; Govoni et al. 2001; Feretti et al. 2004). The intensity of the magnetic field was found to decrease smoothly with the cluster-centric radius, with a trend similar to that of the thermal gas.

From magnetohydrodynamic (MHD) cosmological simulations, an important characterization of the cluster magnetic distribution was made by Dolag et al. (1999, 2002). They studied the correlation between X-ray surface brightness and Faraday rotation measurements (FRMs) in clusters provided by X-ray and radio observations as well as from models for the ICM. These authors performed cosmological MHD simulations in order to recover the correlation between these quantities. They found a relation between magnetic fields and the gas density of the cluster, suggesting that the cluster magnetic fields may span a wide range of spatial scales with a strength that decreases with distance from the cluster center. Murgia et al. (2004) used numerical simulations to investigate the relation between magnetic fields and Faraday rotation effects in clusters. These latter authors compared their simulations with polarization properties of extended cluster radio sources in radio galaxies and halos. They considered that the intensity of magnetic fields decreases from the cluster center in agreement with previous results (Dolag et al. 1999, 2002).

Dolag et al. (1999) found that the observed intra-cluster magnetic field can be reproduced by the evolution of an initial magnetic field at redshift 15 that was amplified by compression during the cluster collapse. One of their important results was that the intra-cluster magnetic field strength is proportional to the gas density at any point $(B(r) \propto \rho(r))$.

Colafrancesco \& Giordano (2007) studied the influence of magnetic fields on the main structural properties of virialized groups and clusters, assuming that it scales with a density of $B(r) \propto \rho^{\alpha}(r)$, as previously proposed. The same power law dependence on the density was used by Zhang (2004) and Koch et al. (2003) to estimate the effect of the intra-cluster magnetic field on the Sunyaev-Zel'dovich power spectrum.

Motivated by these previous works, we assumed a parametric form for the radial distribution of the magnetic field

$B(r)=B_{0}\left(\frac{\rho_{\mathrm{g}}(r)}{\rho_{0}}\right)^{\alpha}$,

where $B_{0}$ is the central value of the magnetic field and $\alpha$ is the shape parameter. Unfortunately, there are no measurements for the magnetic profile for any of the clusters in our sample. Thus, we have to use results from the literature to constrain $B_{0}$ and $\alpha$.

The effective strength and structure of these magnetic fields provide the main challenge, because different methods of analysis give different values for magnetic strength. An estimated consideration of the equipartition of the magnetic field strength averaged over the entire halo volume gives magnetic field strengths of $\sim 0.1-1 \mu \mathrm{G}$ (Govoni et al. 2001; Murgia et al. 2004, and references therein).

Feretti et al. (1999a) estimated that the magnetic field in the ICM of A119 should range between 5-10 $\mu \mathrm{G}$. Bagchi et al. (1998) found $B \approx 1 \mu \mathrm{G}$ for the cluster-scale magnetic field strength. In a more recent work, Clarke et al. (2001) studied a sample of 16 "normal" low-redshift $(z<0.1)$ galaxy clusters, finding that the ICM is permeated with magnetic fields at levels of 4-8 $\mu \mathrm{G}$. Taylor \& Perley (1993) found higher central values, $B \sim 6-30 \mu \mathrm{G}$, for the ICM magnetic fields. Allen et al. (2001) claimed that the central value of the magnetic fields can be $B=12 \mu \mathrm{G}$. FMRs of radio sources provide magnetic fields of $\sim 5-30 \mu \mathrm{G}$ in cooling flow clusters (e.g., 3C 295, Allen et al. 2001; Coma, Feretti et al. 1995; and Hydra A, Taylor \& Perley 1993) where extremely high FRMs have been revealed. Carilli \& Taylor (2002) affirmed that its strength in the center of coolingcore clusters can reach levels of $10-40 \mu \mathrm{G}$.

On the other hand, lower magnetic fields $(\sim 2-8 \mu \mathrm{G})$ have also been detected in clusters without cooling flows (e.g., Feretti et al. 1999b; Taylor et al. 2001; Eilek \& Owen 2002).

The magnetic field strengths obtained from FRMs arguments are higher than the values derived either from the radio data or from inverse Compton X-ray emission. The values deduced from radio synchrotron emission and from inverse Compton refer to averages over large volumes. Instead, FRMs estimates give a weighted average of the field and gas density along the line of sight and could be sensitive to the presence of filamentary structure in the cluster. They could therefore be higher than the average cluster value. However, as pointed out by Carilli \& Taylor (2002), all of these techniques are based on several assumptions. For example, the observed FRMs have been interpreted until now in terms of simple analytical models which consider single-scale magnetic fields, while equipartition calculations in radio halos assume spatially uniform magnetic fields.

There are not many works that studied the power spectrum of the intra-cluster magnetic field fluctuations. However, Enßlin \& Vogt (2003) and Vogt \& Enßlin $(2003,2005)$ by using a new semi-analytical technique showed that, for those cluster sources for which a very detailed FRM image is available, the magnetic field power spectrum can be estimated.

To take into account all these observational results, we considered conservative values in our analysis, adopting for cooling core clusters values for $B_{0}$ ranging from a low value of $5 \mu \mathrm{G}$ up to a strong value of $30 \mu \mathrm{G}$. But for non-cooling core clusters we considered the central strength of the magnetic field to vary from $2 \mu \mathrm{G}<B_{0}<8 \mu \mathrm{G}$. The values adopted agree with both observations and with numerical simulations that considered higher values for magnetic fields in the core of the cluster and a decrease towards the outskirts.

If the ratio of magnetic and thermal pressure is constant throughout the cluster, then $\alpha=0.5$, if we have an homogeneous seed magnetic field compressed during cluster formation, one expects $\alpha=2 / 3$. Vogt \& Enßlin (2005) performed a Bayesian maximum likelihood analysis of Faraday rotation measure in order to derive a power spectrum of cluster magnetic fields. In their analysis they used three different values for $\alpha=0.1,0.5,1.0$. They concluded that values of $\alpha=1.0$ are unlikely, but models with $\alpha=0.1-0.5$ match very well with their calculations. From observational results, Dolag et al. (2001b) found $\alpha=0.9$ for A119 and $\alpha=0.5$ for 3C 129 . If one combines the measurements of the four clusters the data analysis performed by these latter authors leads to a slightly lower slope, of this correlation ( $\alpha=0.8$ ). Therefore, to be conservative, we decide to vary the shape parameter within the interval of $0.5<\alpha<0.9$ to take into account all the above results.

In Fig. 1 we show the mass profile variation due to the magnetic pressure as the only non-thermal component compared to the hydrostatic mass profile. From this Figure we see that the magnetohydrostatic profiles for NCC clusters (A2050 and A12631) present little difference when compared to the hydrostatic profile. This little influence on the magnetic pressure in NCC cluster is due to the central value assumed for these clusters. As the central strength of the magnetic field is lower in NCC clusters, the influence of the magnetic pressure is also lower compared to a CC cluster. For CC clusters we note that 

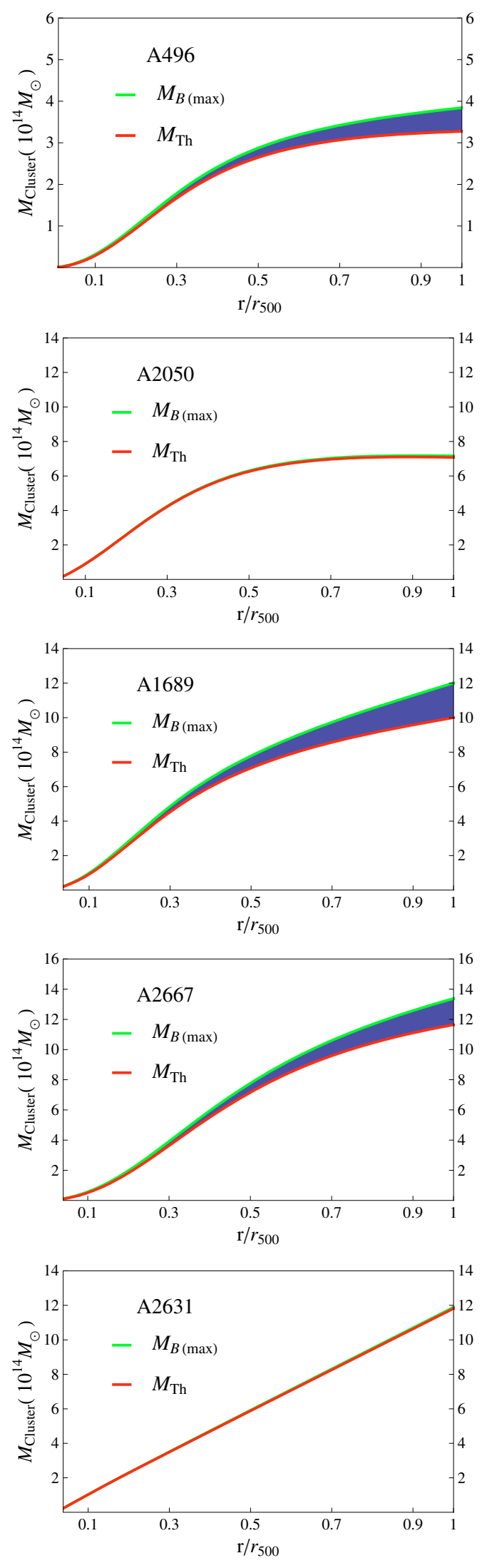

Fig. 1. Variation in the mass profile due to the magnetic pressure as the only non-thermal component for the clusters in our sample. The red lines represent the hydrostatic mass profiles, while the green lines show the maximum variation of mass profiles due to the contribution of the magnetic pressure. The blue zone represents the region of mass profile variation depending on the central value strength $\left(B_{0}\right)$ and the shape parameter $\alpha$. the difference between the hydrostatic and the magnetohydrostatic profiles becomes more pronounced for large radii, that is for $r>0.5 r_{500}$.

Churazov et al. (2008) measured the contribution of the nonthermal pressure in two early-type galaxies that reside in the center of two nearby cool core clusters (Virgo and Fornax). With a similar approach, these authors considered the contribution of all non-thermal components in the same system, combining the contribution of cosmic rays, magnetic fields and microturbulence to the total pressure. They suggested that the $P_{\mathrm{NT}}$ component can account for $10 \%$ of the gas thermal pressure in the core of these galaxies (NGC 1399 and M 87). Assuming that the magnetic component is the only non-thermal pressure, Churazov et al. (2008) constrained the upper limits on magnetic field to be $\sim 20-30 \mu \mathrm{G}$. Besides, Ajello et al. (2009) reported the detection of ten merging-clusters in the $15-55 \mathrm{keV}$ energy band using Swift's Burst Alert Telescope (BAT $\left.{ }^{1}\right)$. These authors coupled radio synchrotron emission (inverse Compton) with X-ray data, putting the constraints on the lower limit of the magnetic field to be typically between $0.1-0.5 \mu \mathrm{G}$. We can see from these Papers mentioned above that both the upper and lower limits adopted in this work are in accordance with the recent literature.

\subsection{Turbulence in clusters of galaxies}

With the advent of high-resolution observations from the Chandra and XMM-Newton satellites, temperature maps of the $\mathrm{X}$-ray emitting gas have shown that even apparently relaxed clusters could have small substructures as imprints of recent minor-mergers (e.g., Finoguenov et al. 2005; Durret \& Lima Neto 2008).

It is widely accepted that the ICM is probably turbulent, and mergers of galactic sub-clusters may be one of the major energy injection mechanisms (see Sarazin 2002; Brunetti 2003; Lazarian 2006, and references therein). Random gas motions can also maintain and amplify cluster magnetic fields via dynamo processes (Roettiger et al. 1999; Subramanian et al. 2006) and contribute to the acceleration of cosmic rays in the ICM (Brunetti \& Lazarian 2007).

Generally the models assume a scenario with the scale for the injection of energy of $100-500 \mathrm{kpc}$ and the injection velocity of the order of $10^{3} \mathrm{~km} \mathrm{~s}^{-1}$.

Since the rate of dissipation of the turbulent energy cannot exceed the X-ray luminosity of the cluster $\left(L_{X}\right)$ in a steady-state, i.e., $\frac{1}{2} v_{0}^{3} / l_{0} \lesssim L_{\mathrm{X}} / M_{\mathrm{g}}$, where $v_{0}$ and $l_{0}$ are the turbulent speed and the scale respectively, and $M_{\mathrm{g}}$ is the gas mass, we have an upper limit on the turbulent velocity as follows (Subramanian et al. 2006)

$v_{0} \lesssim 180 \frac{\mathrm{km}}{\mathrm{s}}\left(\frac{l_{0}}{200 \mathrm{kpc}}\right)^{\frac{1}{3}}\left(\frac{L_{\mathrm{X}}}{10^{45} \mathrm{erg} / \mathrm{s}}\right)^{\frac{1}{3}}\left(\frac{M_{\mathrm{g}}}{10^{14} M_{\odot}}\right)^{\frac{1}{3}}$.

Norman \& Bryan (1999) found that the ICM becomes turbulent during cluster formation, with turbulent velocities of about $400 \mathrm{~km} \mathrm{~s}^{-1}$ within $1 \mathrm{Mpc}$ from the center of a cluster and eddy sizes ranging from 50 to $500 \mathrm{kpc}$. In the cluster merger model of Ricker \& Sarazin (2001), they found large-scale turbulence with eddy sizes up to several hundred kiloparsecs and turbulent velocities of $\sim 100-400 \mathrm{~km} \mathrm{~s}^{-1}$.

From the analysis of pressure fluctuations as revealed in X-ray observations, Schuecker et al. (2004) argue that the

\footnotetext{
${ }^{1}$ http://swift.gsfc.nasa.gov/docs/swift/about_swift/ bat_desc.html
} 
integral turbulent scale in the Coma cluster is close to $100 \mathrm{kpc}$, and they assume a turbulent speed of $250 \mathrm{~km} \mathrm{~s}^{-1}$ at that scale.

Although a number of other studies have examined random gas motions and their effect on the mass estimate (e.g., Rasia et al. 2004; Dolag et al. 2005; Rasia et al. 2006), most of them have used simulations with SPH gas dynamics. The magnitude and effects of gas motions in such simulations depends on the specific treatment of artificial viscosity (Dolag et al. 2005). Thus, we used the results from Lau et al. (2009), which employed simulations with Eulerian gas dynamics with very low numerical viscosity. This approach is therefore useful in evaluating possible differences between numerical techniques and systematic theoretical uncertainties.

In order to quantify the importance of pressure support from random gas motions in clusters, we can write the following relation for the isotropic turbulent pressure $P_{\text {turb }}$ (Lau et al. 2009):

$P_{\text {turb }}=\frac{1}{3} \rho_{\mathrm{g}}\left(\sigma_{\mathrm{r}}^{2}+\sigma_{\mathrm{t}}^{2}\right)$,

where $\sigma_{\mathrm{r}}$ and $\sigma_{\mathrm{t}}$ are the radial and tangential dispersion velocity of the intra-cluster gas respectively. For 16 simulated clusters with virial masses within the range of $\left(5 \times 10^{13}-2 \times 10^{15}\right) M_{\odot}$, Lau et al. (2009) found that gas motions contribute up to $~ 5 \%-$ $15 \%$ of the total pressure support in relaxed clusters. Thus, on average the total mass estimate is biased low by about $8 \pm 2 \%$ (at $r_{500}$ ) in relaxed systems and $11 \pm 6 \%$ in unrelaxed systems. These results agree with previous studies (Evrard 1990; Rasia et al. 2004; Nagai et al. 2007; Piffaretti \& Valdarnini 2008), with contributions increasing along the radius.

Note that random gas pressure and its gradient is sensitive to small-scale clumps and any pressure inhomogeneity, and these sources could potentially bias the measurements of the pressure gradient and hence the hydrostatic mass estimate. In order to minimize this bias Lau et al. (2009) removed subhaloes with a mass greater than $10^{12} h^{-1} M_{\odot}$ and the mass within their tidal radius from their calculation (see their paper for further details).

In our analysis, we used radial and tangential velocity dispersion profiles (see Fig. 2) based on the numerical simulation of Lau et al. (2009). We show these profiles in Fig. 2 where the velocity

$V_{500}=\sqrt{\frac{G M}{r_{500}}}$

is the circular velocity at $r_{500}$.

In Fig. 3 we show the mass profile variation due to the turbulent pressure as the only non-thermal component, compared to the hydrostatic mass profile. From this Figure we see that the influence of the turbulent pressure in the mass estimates is small regardless of weather the cluster is a non-cool core or cool core cluster. Our sample has masses within the range of $(0.3-12) \times 10^{14} M_{\odot}$, and we found that our mass estimates can be biased low by about $\sim 5 \%$.

\subsection{Cosmic ray pressure}

Cosmic ray protons can play an important role within ICM contributing to the equilibrium on the pressure support. Cosmic ray protons (CRp) and cosmic ray electrons (CRe) can be injected into the ICM by three different processes which produce morphologically different radio signature (Brunetti 2003; Pfrommer \& Enßlin 2004):

- shock acceleration: natural acceleration mechanism such as structure formation and merger shocks (Ptuskin et al. 2009; Ostrowski \& Siemieniec-Ozieblo 2002);
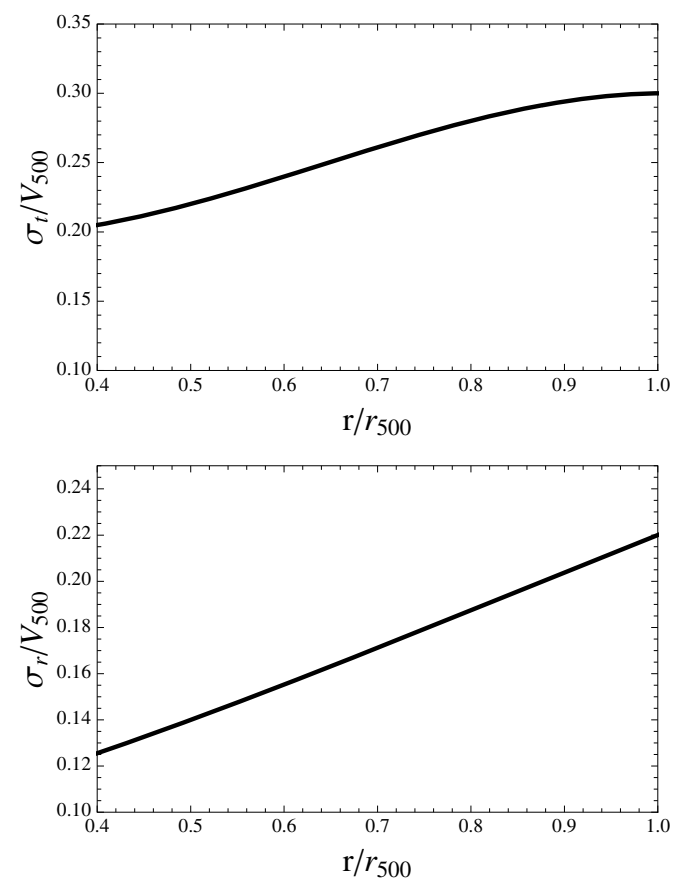

Fig. 2. Velocity dispersion profiles derived from numerical simulation of Lau et al. (2009). Upper panel: tangential velocity profile used to estimate the turbulent pressure. Lower panel: radial velocity profile used to estimate the turbulent pressure.

- reaccelerated electrons: reaccelerated relativistic particles injected by sources like radio galaxies, supernova remnants, merger shocks, galactic winds, etc. (Ensslin et al. 1997; Berezinsky et al. 1997; Pfrommer \& Enßlin 2004);

- particles of hadronic origin: CRp can eventually interact with the thermal ambient gas producing secondary electrons, neutrinos and $\gamma$-rays in an inelastic collision which generates a radio halo through synchrotron emission.

Evidence of non-thermal electrons in the ICM exist in the form of synchrotron radio emission (Feretti \& Giovannini 2008; Brunetti et al. 2008), excess of extreme-ultraviolet (EUV) and hard X-ray radiation (Bowyer \& Berghöfer 1998; Lieu et al. 1999; Fusco-Femiano 2004). Another consequence of the presence of cosmic rays in the ICM is the production of gamma rays.

In order to consider the contribution of cosmic ray pressure to the ICM, we followed the prescription of Ando \& Nagai (2008). We can define a relative contribution of the cosmic ray pressure support as

$Y_{p} \equiv \frac{P_{\mathrm{cr}}}{P_{\mathrm{g}}}$

where this ratio can be parametrized using a power law

$Y_{p}(r)=Y_{p 0}\left(\frac{r}{r_{0}}\right)^{\Psi}$,

where the subscript 0 represents values in the central region. Sijacki et al. (2008), using numerical simulations, followed the evolution of the cosmic-ray inside the bubbles, considering both its hydrodynamical interactions and the dissipation processes relevant to the cosmic ray population. They found that cosmic ray pressure is most relevant in the center of the clusters, being comparable to the thermal pressure for $r<50 h^{-1} \mathrm{kpc}$.

Recent studies have highlighted that CRs can be dynamically important in galaxy clusters because they put constraints 

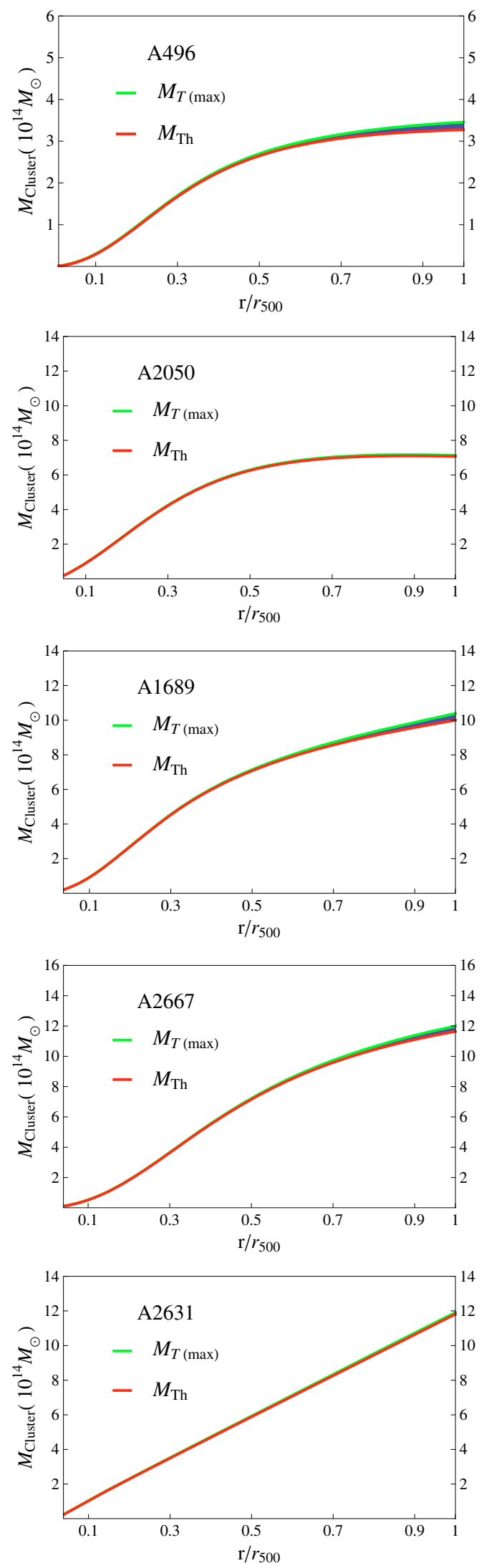

Fig. 3. Variation in the mass profile due to the turbulent pressure as the only non-thermal component for the clusters in our sample. The red line represents the hydrostatic mass profile, while the green line shows the maximum variation of the mass profile due to the contribution of the turbulent pressure. The blue zone represents the region of the mass profile variation depending on the radial and tangential dispersion velocities.
Table 2. Maximum difference in mass estimates.

\begin{tabular}{ccccc}
\hline \hline Cluster & $\sigma_{B}(\max )$ & $\sigma_{\text {turb }}(\max )$ & $\sigma_{\text {cr }}(\max )$ & $\sigma_{\text {total }}(\max )$ \\
\hline A496 & $17.33 \%$ & $5.52 \%$ & $4.87 \%$ & $27.72 \%$ \\
A1689 & $20.07 \%$ & $3.87 \%$ & $9.47 \%$ & $33.40 \%$ \\
A2050 & $1.24 \%$ & $0.82 \%$ & $10.69 \%$ & $12.74 \%$ \\
A2631 & $0.59 \%$ & $0.77 \%$ & $9.79 \%$ & $11.15 \%$ \\
A2667 & $14.92 \%$ & $3.06 \%$ & $9.93 \%$ & $27.90 \%$ \\
\hline
\end{tabular}

on the fraction of cosmic-ray pressure with respect to the thermal pressure $\left(Y_{p}\right)$. Since the Energetic Gamma Ray Experiment Telescope $\left(\mathrm{EGRET}^{2}\right)$ did not detect $\gamma$-ray emission from clusters in the GeV band (Reimer et al. 2003), constraints on the fraction of cosmic ray pressure have been placed in cosmological simulations of the large scale structure. In nearby rich clusters, this component should amount to about $\sim 10-26 \%$ of thermal pressure (Ensslin et al. 1997; Miniati et al. 2001; Miniati 2003). By comparing the integrated $\gamma$-ray flux above $100 \mathrm{MeV}$ to EGRET upper limits, Pfrommer \& Enßlin (2004) constrained the CRp scaling parameter in their simulation of nearby coolingflow clusters. Thus they were able to infer that the $P_{\text {cr }}$ accounts for less than $30 \%$ of the thermal pressure. But Sijacki et al. (2008) affirmed that this component can reach up to $50 \%$ of the central gas pressure in clusters.

Despite all the effort in computing the cosmic ray pressure, the distribution of cosmic-rays in ICM is yet poorly known, and direct evidence for cosmic-ray ions in the ICM is still lacking. The measurements cited above indicate that cosmic ray pressure accounts for a minor contribution to the dynamical support (Ando \& Nagai 2008). We expect that future experiments like the Imaging Air Cherenkov Telescopes (IACTs ${ }^{3}$, which will work in the TeV band) and the Gamma-ray Large Area Space Telescope (GLAST $^{4}$, which will work in the $\mathrm{GeV}$ band) will be able to provide better constraints to $P_{\mathrm{cr}}$ in clusters.

Bearing these results in mind, we used central values for the ratio between the cosmic ray pressure and the thermal pressure between 10-50\%. To which end, we adopted $0.1<Y_{p 0}<0.5$.

The value of $\Psi$ depends on the model adopted for cosmic ray dynamics in clusters. In the simplest model $\Psi=0$, the energy distribution of cosmic rays follows precisely the thermal gas in the cluster. The recent radiative simulations performed by Pfrommer et al. (2007) showed that $\Psi$ takes a value of -0.5 , which was then considered here.

In Fig. 4 we show the mass profile variation due to the cosmic ray pressure as the only non-thermal component, compared to the hydrostatic mass profile. Comparing the results presented in Fig. 1 with Fig. 4 we verify that the cosmic ray pressure is the most important non-thermal component for NCC clusters (see also Table 2).

\section{Mass determination including the effects of non-thermal pressure}

We have assumed that the cluster is spherically symmetric, the gas is in magnetohydrostatic equilibrium and that consequently, the gas pressure and the non-thermal components support the ICM against gravity. We can then write:

$\frac{\mathrm{d}\left(P_{\mathrm{g}}+P_{\mathrm{B}}+P_{\mathrm{turb}}+P_{c r}\right)}{\mathrm{d} r}=-\rho_{\mathrm{g}} \frac{G M_{\mathrm{NTP}}(r)}{r^{2}}$,

\footnotetext{
${ }^{2}$ http://heasarc.gsfc.nasa.gov/docs/cgro/cossc/egret/

3 http://magic.mppmu.mpg.de/introduction/iact.html

${ }^{4}$ http://www-glast.stanford.edu/
} 
T. F. Laganá et al.: Non-thermal pressure in clusters of galaxies
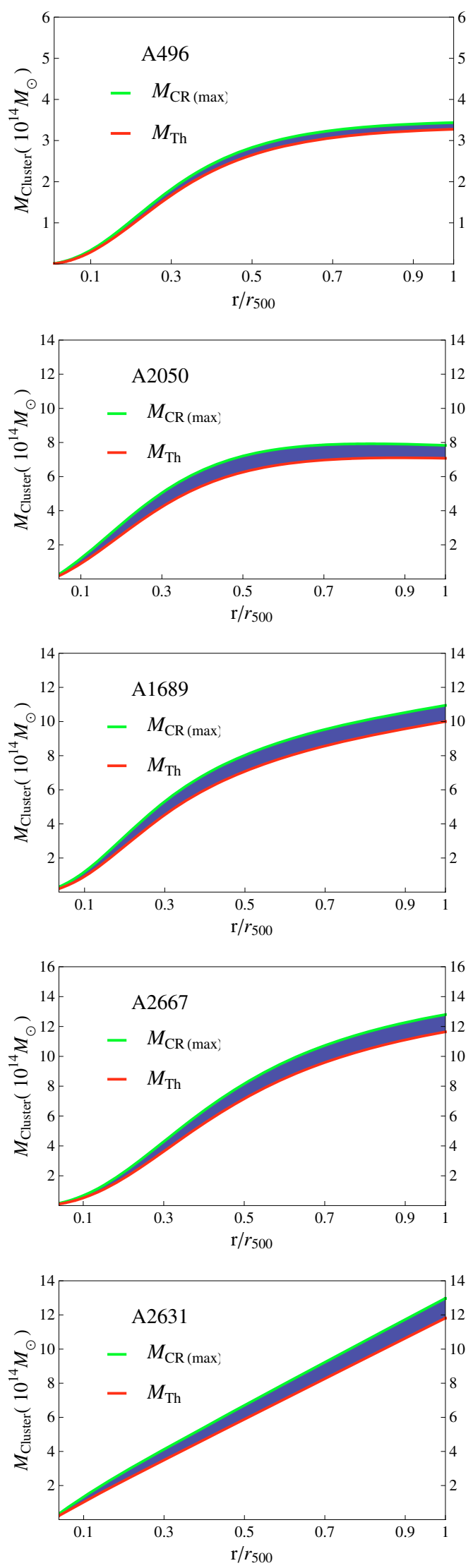

Fig. 4. Variation in the mass profile due to the cosmic ray pressure as the only non-thermal component for the clusters in our sample. The red line represents the hydrostatic mass profile, while the green line shows the maximum variation of the mass profile due to the contribution of the magnetic pressure. The blue zone represents the region of the mass profile variation depending on the $\Psi$ parameter. where the gas pressure $\left(P_{\mathrm{g}}\right)$ at a temperature $T$ is $\rho_{\mathrm{g}} k T / \mu m_{\mathrm{p}}$, the magnetic pressure $\left(P_{B}\right)$ is $\left\langle B^{2}\right\rangle / 8 \pi$, the turbulent pressure $\left(P_{\text {turb }}\right)$ is $\frac{1}{3} \rho_{\mathrm{g}}\left(\sigma_{\mathrm{r}}^{2}+\sigma_{\mathrm{t}}^{2}\right)$, cosmic ray pressure $\left(P_{\mathrm{CR}}\right)$ is $Y_{p 0} P_{\mathrm{g}}\left(r / r_{0}\right)^{\Psi}, G$ is the gravitational constant and $M_{\mathrm{NTP}}$ is the total mass inside a radius $r$. In our case, we computed the total masses within $r_{500}$. Considering the influence of non-thermal pressures in the magnetohydrostatic equilibrium equation, we have the following expression for the total mass of the cluster:

$$
\begin{aligned}
M_{\mathrm{PNT}}(r)= & -\frac{k_{B} T(r)}{G \mu m_{\mathrm{H}}} r\left(\frac{\mathrm{d} \ln \rho_{\mathrm{g}}(r)}{\mathrm{d} \ln r}+\frac{\mathrm{d} \ln T(r)}{\mathrm{d} \ln r}\right) \\
& -\frac{r^{2}}{8 \pi \rho_{\mathrm{g}}(r) G} \frac{\mathrm{d} B(r)^{2}}{\mathrm{~d} r}-\frac{r^{2}}{2 \rho_{\mathrm{g}}(r) G} \frac{\mathrm{d}}{\mathrm{d} r}\left(\rho_{\mathrm{g}}(r) \sigma_{\mathrm{r}}^{2}(r)\right) \\
& -\frac{r}{G}\left(2 \sigma_{\mathrm{r}}^{2}(r)-\sigma_{\mathrm{t}}^{2}(r)\right)-\frac{r^{2}}{G \rho_{\mathrm{g}}(r)} \frac{\mathrm{d} P_{\mathrm{cr}}(r)}{\mathrm{d} r},
\end{aligned}
$$

where $\mu$ is the mean molecular weight, $m_{\mathrm{H}}$ is the hydrogen mass, $k$ is the Boltzmann constant, $T(r)$ is the temperature profile, $B(r)$ is the magnetic profile described in Sect. 3.1, and $\sigma_{\mathrm{r}}$ and $\sigma_{\mathrm{t}}$ are the radial and tangential dispersion velocity of the intra-cluster gas, respectively (see Sect. 3.2).

\section{Results and discussion}

In Fig. 5 we show the evaluation of all non-thermal pressures separately in order to analyze each contribution alone (right panels). The maximum influence which non-thermal components yield in the mass estimate is shown in left panels. From this Figure, we see that the main non-thermal contribution comes from magnetic fields or cosmic rays, depending on the range of parameters adopted.

From this figure we also see that in the inner parts $(r<$ $\sim 0.5 r_{500}$ ) of cool core clusters (A496, A1689 and A2667) the maximum cosmic ray pressure is higher than the maximum magnetic pressure. On the other hand, for the outer parts of the mass profiles the maximum magnetic pressure is always higher than the maximum cosmic ray pressure. However, this statement is not true for non-cool core clusters. For A2050 and A2631, the cosmic ray pressure dominates the magnetic pressure for all radii. Even the turbulent pressure becomes more important than the magnetic component (see Table 2). It is important to note that we used results from the literature to estimate the central magnetic field intensity (e.g., Taylor \& Perley 1993; Feretti et al. 1999a; Allen et al. 2001; Eilek \& Owen 2002) and as observed by Vogt \& Enßlin (2005) the strength of central magnetic fields in non-cool core clusters is lower than those present in cool core clusters. Thus, as the magnetic pressure is described by Eq. (3), a lower central value leads to a decrease in the magnetic pressure.

In Table 2, we present the maximum difference in mass estimates considering the influence of each non-thermal pressure separately and all three components together. We define $\sigma_{B}(\max )$ as the maximum difference in mass estimates due to the magnetic pressure only, $\sigma_{\text {turb }}(\max )$ is the maximum difference in mass estimates due to the turbulence pressure only, $\sigma_{\mathrm{cr}}(\max )$ maximum difference in mass estimates due to the cosmic ray pressure only and $\sigma_{\text {total }}(\max )$ is the maximum difference in mass estimates due to all non-thermal components. The mass variation given by $\sigma M_{\mathrm{NTP}(B)}$ is simply

$\sigma M_{\mathrm{NTP}}=\frac{M_{\mathrm{NTP}}(r)-M(r)}{M(r)}$.

From Table 2, we see that for CC clusters the magnetic pressure is dominant, contributing more than $50 \%$ of the total mass variation. Still, for NCC clusters the major component is the cosmic 

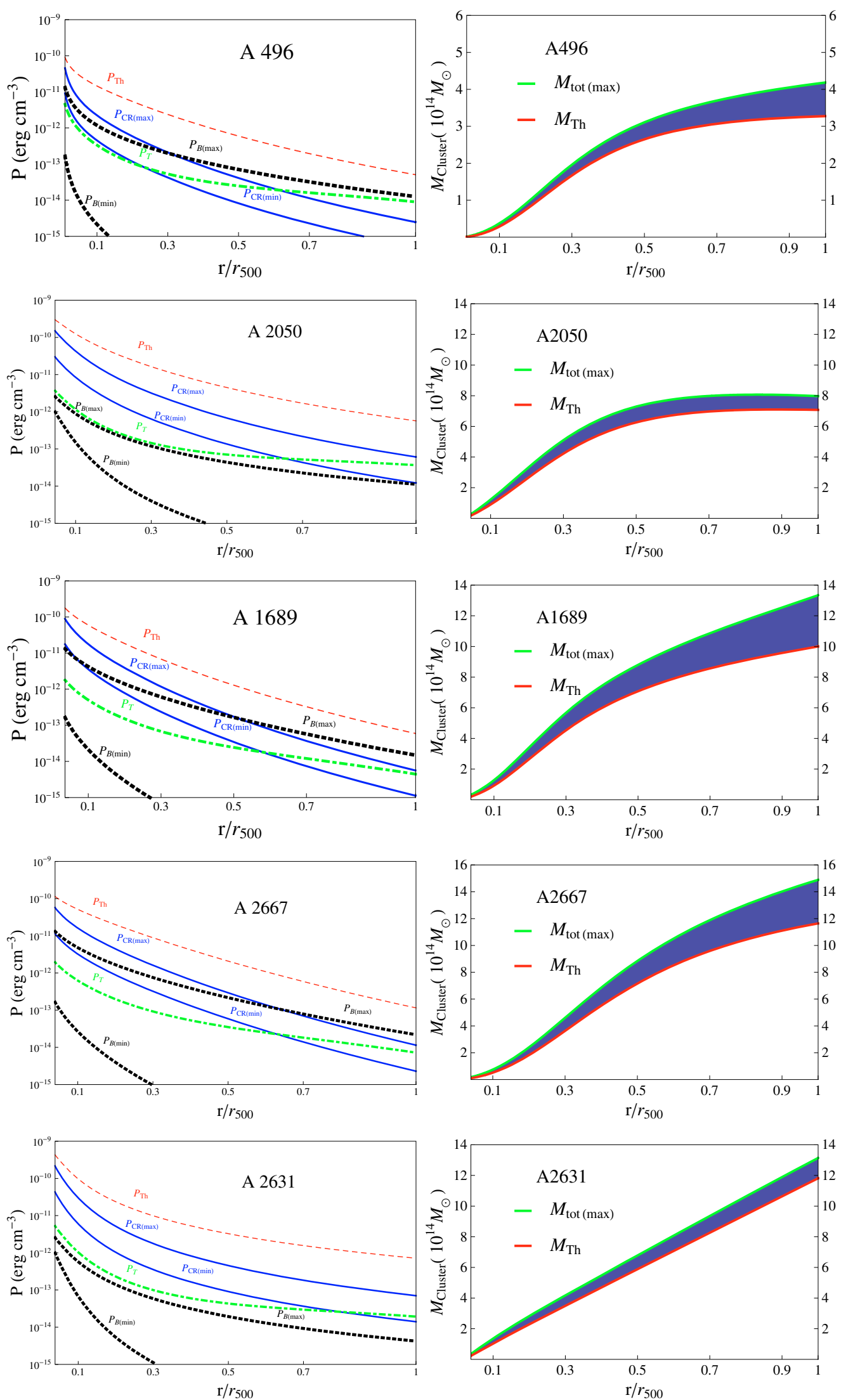

Fig. 5. Left panels: minimum and maximum non-thermal pressure profiles for each component. The red dashed line represents the thermal pressure profile. For the non-thermal pressures, the blue continuous lines represent the maximum and the minimum cosmic ray pressure profiles, the thick black dashed lines represent the maximum and minimum magnetic pressure profiles and the dot-dashed green line represents the turbulent pressure profiles. Right panels: comparison between the hydrostatic mass profile (red lines) and the maximum mass profile due to non-thermal components. The blue region represents all mass profile variations due to all the combinations possible for the three non-thermal components. The clusters are displayed from top to bottom in the following sequence: A496, A2050, A1689, A2667 and A2631. 
ray pressure, accounting for more than $80 \%$ of the total nonthermal pressure. We conclude that the maximum influence of non-thermal components on the total mass variation ranges from more than $10 \%$ to almost $35 \%$. Although all of the assumptions agree with previous works, we emphasize that the difference in the hydrostatic mass estimates rely on the assumption of the specific parametrization of non-thermal components that were inferred based on numerical simulations and observational results. As the errors in mass estimates (by weak lensing or via hydrostatic equilibrium) are in most cases lower than the variation in mass due to non-thermal pressure contribution, we cannot neglect this component. Moreover, as X-ray data are widely used to constrain cosmological parameters, this evaluation should be regarded with care. Since in most cases this method neglects the non-thermal contribution to mass estimates, these constraints may be biased low and consequently it will bias the cosmological determinations. Thus, multi-wavelength study will play an important hole in the investigation of non-thermal components in galaxy clusters.

\subsection{Constraining the non-thermal pressure based on A1689}

The plasma in many apparently relaxed systems may be affected by additional non-equilibrium processes, which contribute to rise the pressure and hence cause an underestimate of the cluster mass from X-ray observations of the thermal bremsstrahlung emission. If this difference is due to non-thermal pressure, the comparison between independent methods of mass estimates can provide a powerful constraint to the contribution of this component.

It is important to notice that weak lensing study is only possible if the cluster is massive and not very close. For the sample chosen in this work, the only cluster with available weaklensing mass determination inside $r_{500}$ is A1689. In this section we focus on the comparison of a hydrostatic X-ray mass estimate determined in a previous work (Laganá et al. 2008) with those derived from weak lensing which are available in the literature. However, as noted by Hoekstra (2007), it is difficult to compare results from different mass indicators since it would be necessary to make assumptions regarding the cluster geometry in the $\mathrm{X}$-ray determination, and because lensing is sensitive to all mass along the line of sight, this would also bias weak lensing mass determination.

The total mass inside $r_{500}$ computed from X-ray measurements is $M_{500}=(11.14 \pm 0.46) \times 10^{14} M_{\odot}$ without considering the non-thermal component. For A1689, Mahdavi et al. (2008) estimated a total mass inside $r_{500}$ of $M_{500}=(14.29 \pm 2.40) \times 10^{14} M_{\odot}$. In this case, the total non-thermal contribution (this means all three components: cosmic ray, magnetic and turbulent pressure) to the mass estimates can range from $\sim 2 \%$ to more than $30 \%$.

This cluster is possibly undergoing a merger, where a subclump close to or along the line-of-sight is being accreted (Andersson \& Madejski 2004). Thus, in this specific case, the turbulent motion seems to account for the most part of the nonthermal pressure. Nagai et al. (2007) affirmed that the hydrostatic estimate of the total mass is biased low by about $5-20 \%$ through the virial region, primarily due to additional pressure support provided by subsonic bulk motions in the ICM.

On average, the hydrostatic cluster mass estimates are biased low by about $7.5 \%$ at $r_{500}$ for relaxed systems, while the bias in unrelaxed systems is about $10.5 \%$ at this radius (Lau et al. 2009). From our results (see Fig. 5 for this cluster) we see that if one assume the non-thermal contribution for this cluster, the mass estimates from different methods (e.g., weak lensing and $\mathrm{X}$-rays) can be totally compatible.

\section{Conclusion}

We have taken into account the effects of non-thermal pressure on the X-ray mass estimates for five Abell clusters (A496, A2050, A1689, A2667 and A2631). The masses derived considering just the thermal pressure were presented in a previous work by Laganá et al. (2008) and were used here for comparison. We summarize our main results below:

- The inclusion of non-thermal pressure in the intra-cluster gas description is motivated by the increasing evidence for the presence of a magnetic field in clusters of galaxies. We assume a magnetic profile given by $B(r) \propto B_{0} \rho_{\mathrm{g}}^{\alpha}$, considering values for $B_{0}$ ranging from 5 up to $30 \mu \mathrm{G}$ for CC clusters, while for NCC clusters we assume $2 \mu \mathrm{G}<B_{0}<8 \mu \mathrm{G}$. For each central value we let the shape parameter vary between $0.5<\alpha<0.9$. The magnetic pressure contributes with approximately $20 \%$ to the total mass variation.

- In order to take into account the influence of turbulent motion in the ICM and bulk velocities, we assume isotropic turbulent pressure based on the numerical simulation results of Lau et al. (2009): $P_{\text {turb }}=1 / 3 \rho_{\mathrm{g}}\left(\sigma_{\mathrm{r}}^{2}+\sigma_{\mathrm{t}}^{2}\right)$. The tangential $\left(\sigma_{\mathrm{r}}\right)$ and the radial $\left(\sigma_{\mathrm{t}}\right)$ dispersion velocity profiles were derived based in the same numerical simulations. This component can influence up to $5 \%$ of the total mass estimates.

- Energetic particles are confined by magnetic fields. Since ICM is permeated by magnetic fields, cosmic rays can also provide an important source of pressure. As the distribution of cosmic rays is poorly known, we considered the prescription of Ando \& Nagai (2008) to derive the cosmic ray pressure. From our results we can see that this component can affect the cluster mass estimates by $\sim 10 \%$. In the inner parts of cool core clusters this component is comparable to the magnetic pressure.

- The plasma in clusters of galaxies may be affected by nonthermal processes which rise the pressure and hence cause an underestimate in X-ray measurements of the total mass. In this way the comparison between independent methods of mass estimates can constrain the contribution of nonthermal pressure. We compared weak-lensing results with $\mathrm{X}$-ray mass measurements for A1689 to investigate this, and if the difference in mass estimates is in fact due to the nonthermal component, it can account for $2 \%$ to $\sim 30 \%$.

- We took into account the effects of non-thermal pressure on the total mass estimates. To derive the thermal pressure and compute the hydrostatic mass we used observed temperature and density profiles (Laganá et al. 2008). This is the first study that considers the influence of all three non-thermal pressure on the total mass estimates. Within the limits of our sample and with several reasonable assumptions to describe each of the non-thermal components, it is important to notice that our findings rely on the specific parametrization adopted in this work. This study indicates that further investigations are needed to make a detailed description of the influence of non-thermal components in ICM.

- Without the complete knowledge of the non-thermal contribution, we should be aware of the fact that clusters of galaxies have been used as observational tools for investigations of cosmological interest based only on the hydrostatic assumption. The non-thermal components are neglected. A thorough knowledge of non-thermal contribution will come from the 
combination of X-ray, radio and gamma ray data for a large sample of clusters. The next generation of cluster surveys will provide data to address these fundamental questions in cosmology 5 .

Acknowledgements. We acknowledge Gastão B. Lima Neto, Renato A. Dupke and Elisabete M. De Gouveia Dal Pino for stimulating discussions. We also acknowledge the anonymous referee for the useful suggestions which improved our work. We gratefully thank Florence Durret, Reuven Opher and Emille Ishida for revising this manuscript. This work was support by the Brazilian agency FAPESP (grants: 08/04318-7, 04/05961-0, 06/58240-3).

\section{References}

Ajello, M., Rebusco, P., Cappelluti, N., et al. 2009, ApJ, 690, 367 Allen, S. W., Taylor, G. B., Nulsen, P. E. J., et al. 2001, MNRAS, 324, 842 Andernach, H., Han Tie, Sievers, A., et al. 1988, A\&AS, 73, 265 Andersson, K. E., \& Madejski, G. M. 2004, ApJ, 607, 190

Ando, S., \& Nagai, D. 2008, MNRAS, 385, 2243

Bagchi, J., Pislar, V., \& Lima Neto, G. B. 1998, MNRAS, 296, L23 Berezinsky, V. S., Blasi, P., \& Ptuskin, V. S. 1997, ApJ, 487, 529

Bowyer, S., \& Berghöfer, T. W. 1998, ApJ, 506, 502

Brunetti, G. 2003, ed. S. Bowyer, \& C.-Y. Hwang, ASP Conf. Ser., 301, 349 Brunetti, G., \& Lazarian, A. 2007, MNRAS, 378, 245

Brunetti, G., Setti, G., Feretti, L., \& Giovannini, G. 2001, MNRAS, 320, 365

Brunetti, G., Giacintucci, S., Cassano, R., et al. 2008, Nature, 455, 944

Carilli, C. L., \& Taylor, G. B. 2002, A\&RA, 40, 319

Cavaliere, A., \& Fusco-Femiano, R. 1976, A\&A, 49, 137

Cavaliere, A., \& Fusco-Femiano, R. 1978, A\&A, 70, 677

Churazov, E., Sunyaev, R., Forman, W., \& Böhringer, H. 2002, MNRAS, 332, 729

Churazov, E., Forman, W., Vikhlinin, A., et al. 2008, MNRAS, 388, 1062

Clarke, T. E., Kronberg, P. P., \& Böhringer, H. 2001, ApJ, 547, L111

Colafrancesco, S., \& Giordano, F. 2007, A\&A, 466, 421

David, L. P., Jones, C., \& Forman, W. 1995, ApJ, 445, 578

Demarco, R., Magnard, F., Durret, F., \& Márquez, I. 2003, A\&A, 407, 437

Dolag, K., \& Schindler, S. 2000, A\&A, 364, 491

Dolag, K., Bartelmann, M., \& Lesch, H. 1999, A\&A, 348, 351

Dolag, K., Evrard, A., \& Bartelmann, M. 2001a, A\&A, 369, 36

Dolag, K., Schindler, S., Govoni, F., \& Feretti, L. 2001b, A\&A, 378, 777

Dolag, K., Bartelmann, M., \& Lesch, H. 2002, A\&A, 387, 383

Dolag, K., Vazza, F., Brunetti, G., \& Tormen, G. 2005, MNRAS, 364, 753

Dreher, J. W., Carilli, C. L., \& Perley, R. A. 1987, ApJ, 316, 611

Durret, F., \& Lima Neto, G. B. 2008, Advances in Space Research, 42, 578

Durret, F., Lima Neto, G. B., \& Forman, W. 2005, A\&A, 432, 809

Eilek, J. A., \& Owen, F. N. 2002, ApJ, 567, 202

Ensslin, T. A., Biermann, P. L., Kronberg, P. P., \& Wu, X.-P. 1997, ApJ, 477, 560

Enßlin, T. A., \& Vogt, C. 2003, A\&A, 401, 835

Evrard, A. E. 1990, ApJ, 363, 349

Feretti, L., \& Giovannini, G. 2008, in A Pan-Chromatic View of Clusters of Galaxies and the Large-Scale Structure, ed. M. Plionis, O. López-Cruz, \&

D. Hughes (Berlin: Springer Verlag), Lecture Notes in Physics, 740, 143

Feretti, L., Dallacasa, D., Giovannini, G., \& Tagliani, A. 1995, A\&A, 302, 680 Feretti, L., Dallacasa, D., Govoni, F., et al. 1999a, A\&A, 344, 472

Feretti, L., Perley, R., Giovannini, G., \& Andernach, H. 1999b, A\&A, 341, 29

Feretti, L., Orrù, E., Brunetti, G., et al. 2004, A\&A, 423, 111

Finoguenov, A., Reiprich, T. H., \& Böhringer, H. 2001, A\&A, 368, 749

Finoguenov, A., Böhringer, H., \& Zhang, Y.-Y. 2005, A\&A, 442, 827

Fusco-Femiano, R. 2004, Ap\&SS, 294, 37
Giovannini, G., Feretti, L., Venturi, T., Kim, K.-T., \& Kronberg, P. P. 1993, ApJ, 406, 399

Govoni, F., \& Feretti, L. 2004, Int. J. Mod. Phys. D, 13, 1549

Govoni, F., Enßlin, T. A., Feretti, L., \& Giovannini, G. 2001, A\&A, 369, 441

Hoekstra, H. 2007, MNRAS, 379, 317

Jaffe, W. 1980, ApJ, 241, 925

Jones, C., \& Forman, W. 1984, ApJ, 276, 38

Koch, P. M., Jetzer, P., \& Puy, D. 2003, New Astron., 8, 1

Lacey, C., \& Cole, S. 1993, MNRAS, 262, 627

Laganá, T. F., Lima Neto, G. B., Andrade-Santos, F., \& Cypriano, E. S. 2008, A\&A, 485, 633

Lau, E. T., Kravtsov, A. V., \& Nagai, D. 2009, [arXiv:0903.4895]

Lazarian, A. 2006, Astron. Nachr., 327, 609

Lieu, R., Ip, W.-H., Axford, W. I., \& Bonamente, M. 1999, ApJ, 510, L25

Loeb, A., \& Mao, S. 1994, ApJ, 435, L109

Mahdavi, A., Hoekstra, H., Babul, A., \& Henry, J. P. 2008, MNRAS, 384, 1567

Markevitch, M., \& Vikhlinin, A. 2007, Phys. Rep., 443, 1

Miniati, F. 2003, MNRAS, 342, 1009

Miniati, F., Jones, T. W., Kang, H., \& Ryu, D. 2001, ApJ, 562, 233

Murgia, M., Govoni, F., Feretti, L., et al. 2004, A\&A, 424, 429

Nagai, D., Vikhlinin, A., \& Kravtsov, A. V. 2007, ApJ, 655, 98

Norman, M. L. \& Bryan, G. L. 1999, in The Radio Galaxy Messier 87, ed.

H.-J. Röser, \& K. Meisenheimer (Berlin: Springer Verlag), Lecture Notes in Physics, 530, 106

Ostrowski, M., \& Siemieniec-Ozieblo, G. 2002, A\&A, 386, 829

Perley, R. A., \& Taylor, G. B. 1991, AJ, 101, 1623

Pfrommer, C., \& Enßlin, T. A. 2004, A\&A, 413, 17

Pfrommer, C., Enßlin, T. A., Springel, V., Jubelgas, M., \& Dolag, K. 2007, MNRAS, 378, 385

Piffaretti, R., \& Valdarnini, R. 2008, A\&A, 491, 71

Pislar, V., Durret, F., Gerbal, D., Lima Neto, G. B., \& Slezak, E. 1997, A\&A, 322, 53

Ptuskin, V. S., Rogovaya, S. I., \& Zirakashvili, V. N. 2009, Bulletin of the Russian Academy of Science, Phys., 73, 552

Rasia, E., Tormen, G., \& Moscardini, L. 2004, MNRAS, 351, 237

Rasia, E., Ettori, S., Moscardini, L., et al. 2006, MNRAS, 369, 2013

Reimer, O., Pohl, M., Sreekumar, P., \& Mattox, J. R. 2003, ApJ, 588, 155

Reiprich, T. H., \& Böhringer, H. 2002, ApJ, 567, 716

Ricker, P. M., \& Sarazin, C. L. 2001, ApJ, 561, 621

Roettiger, K., Burns, J. O., \& Stone, J. M. 1999, ApJ, 518, 603

Sánchez-Salcedo, F. J., Brandenburg, A., \& Shukurov, A. 1999, Ap\&SS, 263, 87

Sarazin, C. L. 1986, Rev. Mod. Phys., 58, 1

Sarazin, C. L. 2002, in Merging Processes in Galaxy Clusters, ed. L. Feretti, I. M. Gioia, \& G. Giovannini, Astrophysics and Space Science Library, 272, 1

Schuecker, P., Finoguenov, A., Miniati, F., Böhringer, H., \& Briel, U. G. 2004, A\&A, 426, 387

Sijacki, D., Pfrommer, C., Springel, V., \& Enßlin, T. A. 2008, MNRAS, 387, 1403

Soker, N., \& Sarazin, C. L. 1990, ApJ, 348, 73

Subramanian, K., Shukurov, A., \& Haugen, N. E. L. 2006, MNRAS, 366, 1437

Taylor, G. B., \& Perley, R. A. 1993, ApJ, 416, 554

Taylor, G. B., Barton, E. J., \& Ge, J. 1994, AJ, 107, 1942

Taylor, G. B., Govoni, F., Allen, S. W., \& Fabian, A. C. 2001, MNRAS, 326, 2

Taylor, G. B., Fabian, A. C., \& Allen, S. W. 2002, MNRAS, 334, 769

Vikhlinin, A., Kravtsov, A., Forman, W., et al. 2006, ApJ, 640, 691

Vogt, C., \& Enßlin, T. A. 2003, A\&A, 412, 373

Vogt, C., \& Enßlin, T. A. 2005, A\&A, 434, 67

Voit, G. M. 2005, Rev. Mod. Phys., 77, 207

White, D. A., \& Fabian, A. C. 1995, MNRAS, 273, 72

Xue, Y.-J., \& Wu, X.-P. 2000, MNRAS, 318, 715

Zhang, P. 2004, MNRAS, 348, 1348

$\overline{5}$ http://wfxt.pha.jhu.edu/

Page 10 of 10 